\title{
Stand-Alone Cervical Cages in 2-Level Anterior Interbody Fusion in Cervical Spondylotic Myelopathy: Results from a Minimum 2-Year Follow-up
}

\author{
Eugene Pak-Lin Ng, Andrew Siu-Leung Yip, Keith Hay-Man Wan, Michael Siu-Hei Tse, \\ Kam-Kwong Wong, Tik-Koon Kwok, Wing-Cheung Wong \\ Department of Orthopaedics and Traumatology, Kwong Wah Hospital, Hong Kong
}

\begin{abstract}
Study Design: A retrospective review of patients who underwent 2-level anterior cervical discectomy and fusion (ACDF) with standalone polyetheretherketone (PEEK) cages for cervical spondylotic myelopathy (CSM).

Purpose: To evaluate the efficacy of stand-alone PEEK cage in 2-level cervical interbody fusion for CSM.

Overview of Literature: ACDF is a standard surgical procedure to treat degenerative disc disease. However, the use of additional anterior plating for 2-level ACDF remains controversial.

Methods: We reviewed outcomes of patients who underwent 2-level ACDF with stand-alone PEEK cages for CSM over a 7-year period (2007-2015) in a regional hospital. Japanese Orthopaedic Association (JOA) score, fusion rate, subsidence rate, cage migration, and cervical alignment by the C2-7 angle as well as the local segmental angle (LSA) of the cervical spine were assessed.

Results: In total, 31 patients (mean age, 59 years; range, 36-87 years) underwent 2-level ACDF with a cage-only construct procedure between 2007 and 2015. The minimum follow-up was 24 months; mean follow-up was 51 months. C3-5 fusion was performed in $45 \%$, C4-6 fusion in 32\%, and C5-7 fusion in 23\%. Mean JOA score improved from $10.1 \pm 2.2$ to $13.9 \pm 2.1(p<0.01)$ at the 24 -month follow-up. Fusion was achieved in all patients. Subsidence occurred in $22.5 \%$ of the cages but was not associated with differences in JOA scores, age, sex, or levels fused. Lordosis of the C2-7 angle and LSA increased after surgery, which were maintained for up to 1 year but subsequently disappeared after 2 years, yet the difference was not statistically significant. No cage migration was noted; two patients developed adjacent segment disease requiring posterior laminoplasty 3 years after ACDF.

Conclusions: The use of a stand-alone PEEK cage in a 2-level cervical interbody fusion achieves satisfactory improvements in both clinical outcomes and fusion.
\end{abstract}

Keywords: Cervical spondylotic myelopathy; Anterior cervical discectomy and fusion; Stand-alone cage; Two level fusion

\section{Introduction}

Anterior cervical discectomy and fusion (ACDF) is a well- established treatment for cervical degenerative disc disease, which was first described by Smith and Robinson in 1958 [1]. It aims to decompress the spinal cord and nerve

\footnotetext{
Received Aug 2, 2018; Revised Aug 26, 2018; Accepted Sep 6, 2018

Corresponding author: Eugene Pak-Lin Ng

Department of Orthopaedics \& Traumatology, Kwong Wah Hospital, 25 Waterloo Road, Kowloon, Hong Kong

Tel: +852-35177877, Fax: +852-35177201, E-mail: npl566@ha.org.hk
} 
roots, restore lordosis, and stabilize spinal segments. Unfortunately, the use of autologous iliac bone grafts and anterior cervical plating has been associated with donor site morbidity and dysphagia, respectively $[2,3]$. As a result, intervertebral cages were introduced to reduce the morbidity associated with iliac bone grafts, and they are designed to restore lordosis, preserve disc height, and prevent implant collapse [4]. Interbody fusion using a standalone cage without anterior cervical plating has been shown to reduce complications, generate less dysphagia [5], and yet achieve similar radiological and clinical outcomes [6], all in single-level fusions. Currently, however, there is no strong evidence establishing the safety and efficacy of stand-alone cages in 2-level ACDF procedures. Therefore, in this study, we retrospectively reviewed the clinical and radiological outcomes of 2-level ACDF with stand-alone polyetheretherketone (PEEK) cages in treating cervical spondylotic myelopathy (CSM), with a minimum of 2 years of follow-up.

\section{Materials and Methods}

This was a retrospective review of patients with CSM who underwent 2-level ACDF with stand-alone PEEK cages filled with bone substitute, in Kwong Wah Hospital, Hong Kong, from January 2007 to May This retrospective study was exempted from application of the institutional review board approval in Kwong Wah Hospital, Hong Kong. Indications for 2-level ACDF included patients with CSM who had the presence of neurological deficits confirmed by magnetic resonance imaging (MRI). Neurological deficits were defined as motor weakness; sensory deficits; or the presence of myelopathic hand signs, trunk ataxia, or lower limb spasticity on physical examination. Features on MRI confirming CSM included definite stenosis with cord impingement, or compression with or without the presence of a hyperintense cord signal on the T2-weighted sequence. The inclusion criterion for this review was 2-level ACDF for adjacent segments. Patients who received separate 2-level ACDF were excluded. All operations were performed by two of the four in-house spine surgeons. A left-sided standard anterior approach to the cervical spine, with cervical discectomy was performed with the aid of an operating microscope. The posterior longitudinal ligament was excised to decompress the dura, and the cartilaginous endplates were excised. Interbody fusion was achieved with a self-locking PEEK cage (Cervios;
DePuy Synthes, Zuchwil, Switzerland). Beta-tricalcium phosphate synthetic bone substitute (ChronOs, DePuy Synthes) was placed within the cage to aid fusion. A soft neck collar was prescribed for 2-4 weeks after the surgery, for sitting and walking, but was not required in bed.

\section{Clinical parameters}

The Japanese Orthopaedic Association (JOA) score was measured preoperatively and during subsequent postoperative follow-ups at 3 months, 6 months, 1 year, and then annually. A full score was 17 points, with 4 points each for upper and lower limb motor function, 6 points for limb and trunk sensation, and 3 for bladder and bowel function. The recovery rate for the JOA score was calculated using Hirabayashi's method: recovery rate $(\%)=$ (postoperative JOA-preoperative JOA)/(17-preoperative JOA) $\times 100$.

\section{Radiological parameters}

Anteroposterior and lateral cervical spine radiographs were taken preoperatively (with flexion and extension) and immediately postoperatively, as well as at 3 months, 6 months, 1 year, and then annually. Radiological parameters assessed in this study included fusion, cage subsidence, presence of cage migration, and cervical spine sagittal alignment. Radiographs were reviewed by two independent surgeons (Ng and Wan). Discrepancies were resolved after discussion in the presence of a third surgeon (Yip).

Fusion was evaluated according to Peolsson's classification using the 1-year follow-up radiograph [7]: type 1A fusion denoted the presence of bridging bone anterior to and through the disc space; type $1 \mathrm{~B}$ fusion denoted the presence of bridging bone anterior to but not through the disc space; type $2 \mathrm{~A}$ fusion denoted the presence of bridging bone through the disc space only; and type $2 \mathrm{~B}$ fusion denoted the absence of bridging bone.

Subsidence was defined as any change of at least $3 \mathrm{~mm}$ in the anterior disc space height, posterior disc space height, or a posterior migration of the cage when compared to the preoperative radiograph at each time point [8]. Migration was defined as any change in the anteroposterior cage position when compared to the preoperative radiograph at each time point. Clinical implications of cage subsidence were investigated by comparing mean 
differences in the JOA score between patients with or without cage subsidence.

Cervical sagittal alignment was measured using Cobb's angle, which was calculated using two lines drawn parallel to the inferior endplates of the $\mathrm{C} 2$ and $\mathrm{C} 7$ vertebral bodies (Fig. 1). Local segmental alignment was measured using Cobb's angle formed between a line parallel to the superior endplate of the vertebra above the involved disc levels and a line parallel to the inferior endplate of the vertebra below the involved disc levels. Focal segmental kyphosis was defined as an increase of more than $5^{\circ}$ in kyphosis at the 2-year follow-up, compared to the initial postoperative film.

\section{Statistical techniques}

All analyses were conducted with the SPSS SPSS for Windows ver. 16.0 (SPSS Inc., Chicago, IL, USA). Basic data
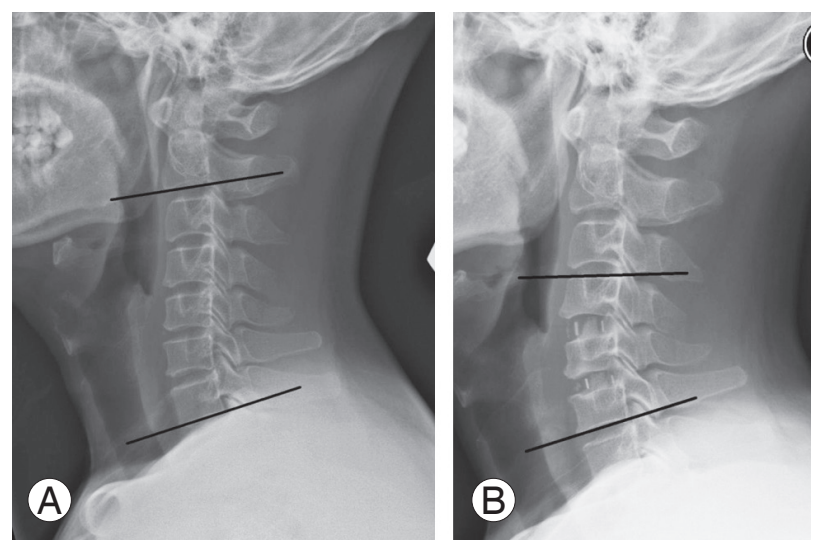

Fig. 1. Cervical alignment. (A) Cervical spine alignment measured by Cobb's angle between the inferior endplate of the $\mathrm{C} 2$ body and the inferior endplate of the C7 body. (B) Local segmental angle of fused segments measured by Cobb's angle between the superior endplate of the upper vertebra and the inferior endplate of the lower vertebra. were presented as the mean and standard deviation for continuous variables (e.g., JOA score, C2-7 angle, local segmental angle [LSA], etc.), and as percentages for categorical variables (e.g., subsidence). Paired $t$-tests were used to compare continuous variables before and after ACDF. Independent sample t-tests were used to compare continuous variables (age, JOA score, $\mathrm{C} 2-7$ angle, LSA) between those with or without subsidence. The Pearson chi-square test or Fisher's exact test was used to assess the association between categorical variables (e.g., sex, subsidence, levels of ACDF). A $p$-value of $<0.05$ was considered statistically significant, and all tests were two-tailed.

\section{Results}

Thirty-one patients received 2-level ACDF for CSM using stand-alone cages in the period from January 2007 to May 2015. All patients were followed up for at least 2 years; the mean follow-up time in this study was 51 months. The patients' demographics are detailed in Table 1. Most patients

Table 1. Baseline characteristics

\begin{tabular}{lc} 
Characteristic & Value \\
Sex & \\
$\quad$ Male & $25(81)$ \\
Female & $6(9)$ \\
Age (yr) & $59.7 \pm 12$ \\
\hline Levels of anterior cervical discectomy and fusion & \\
\hline C3-5 & $14(45)$ \\
C4-6 & $10(32)$ \\
C5-7 & $7(23)$ \\
\hline
\end{tabular}

Values are presented as number (\%) or mean \pm standard deviation.

Table 2. Clinical and radiological outcomes

\begin{tabular}{|c|c|c|c|c|c|}
\hline \multirow{2}{*}{ Variable } & \multirow{2}{*}{ Preoperative } & \multicolumn{4}{|c|}{ Duration of follow-up } \\
\hline & & $3 \mathrm{mo}$ & $6 \mathrm{mo}$ & $1 \mathrm{yr}$ & $2 y r$ \\
\hline \multicolumn{6}{|l|}{ Clinical outcomes } \\
\hline Japanese Orthopaedic Association score & $10.1 \pm 2.2$ & $13.1 \pm 2.0^{*}$ & $13.3 \pm 2.1^{*}$ & $13.3 \pm 2.2^{*}$ & $13.9 \pm 2.1^{*}$ \\
\hline \multicolumn{6}{|l|}{ Radiological outcomes } \\
\hline Subsidence & - & 0 & $14(22.5)$ & $14(22.5)$ & $14(22.5)$ \\
\hline C2-7 angle $\left(^{\circ}\right)$ & $8.19 \pm 13.2$ & $10.7 \pm 11.5$ & $11.5 \pm 14.1$ & $13.0 \pm 15.3$ & $10.9 \pm 14.5$ \\
\hline Local segmental angle $\left(^{\circ}\right)$ & $4.44 \pm 8.91$ & $5.56 \pm 6.79$ & $5.76 \pm 8.61$ & $5.29 \pm 10.3$ & $3.06 \pm 9.03$ \\
\hline
\end{tabular}

Values are presented as mean \pm standard deviation or number (\%).

${ }^{*} p<0.01$ comparing mean differences against preoperative value. 


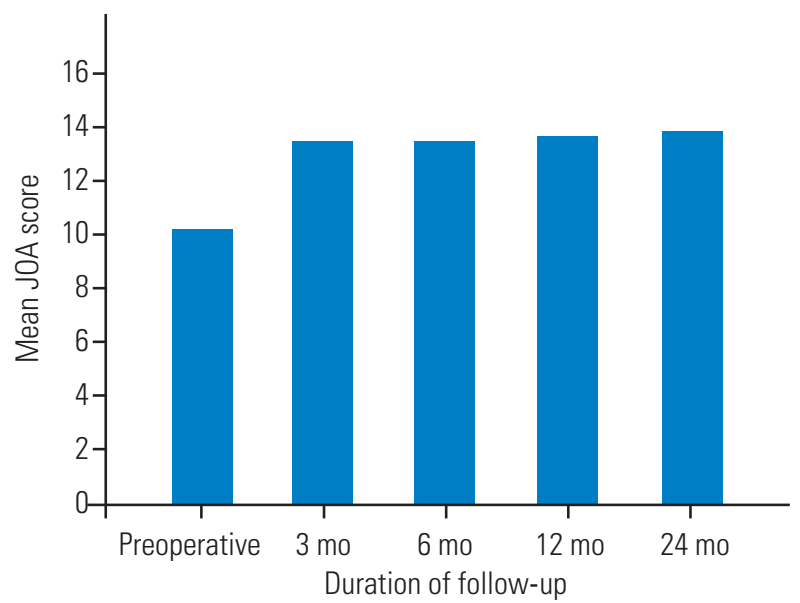

Fig. 2. JOA score. JOA, Japanese Orthopaedic Association.

were male $(\mathrm{n}=25)$ and the mean age was 60 years (range, $36-87$ years). Surgery was most commonly performed in levels C3-5 ( $\mathrm{n}=14)$, followed by C4-6 $(\mathrm{n}=10)$, and lastly in $\mathrm{C} 5-7(\mathrm{n}=7)$.

\section{Clinical outcomes}

At baseline, the mean JOA score was $10.1 \pm 2.2$. The value increased to $13.1 \pm 2.0(p<0.01)$ at the 3-month follow-up. The mean recovery rate calculated using Hirabayashi's method was $43.9 \%$. Subsequently, the mean JOA scores at 6 months, 1 year, and 2 years were $13.3 \pm 2.1(p<0.01)$, $13.3 \pm 2.2(p<0.01)$, and $13.9 \pm 2.1(p<0.01)$, respectively (Table 2, Fig. 2). The JOA scores were maintained after the ACDF procedures, with no statistically significant differences when comparing scores at 3 months, 6 months, 1 year, and 2 years of follow-up.

Two patients (6.4\%) developed adjacent segment disease (ASD) requiring posterior laminoplasty. One patient who initially underwent a C3-C5 ACDF developed ASD at the C5-6 level 2 years later and subsequently underwent C3C6 laminoplasty. This patient did not have congenital canal stenosis and the canal anteroposterior diameters were $12.1 \mathrm{~mm}$ at $\mathrm{C} 3,12.2 \mathrm{~mm}$ at $\mathrm{C} 4$, and $12.5 \mathrm{~mm}$ at $\mathrm{C} 5$.

Another patient who underwent a C4-C6 ACDF developed ASD at the C3-4 level 3 years later, which required a C3-C6 laminoplasty (Fig. 3). In this case, there was no congenital canal stenosis and the canal diameters were $11.4 \mathrm{~mm}$ at $\mathrm{C} 4,11.8 \mathrm{~mm}$ at $\mathrm{C} 5$, and $12.1 \mathrm{~mm}$ at $\mathrm{C} 6$.

\section{Radiological outcomes}

All patients achieved radiological fusion at 12 months. In
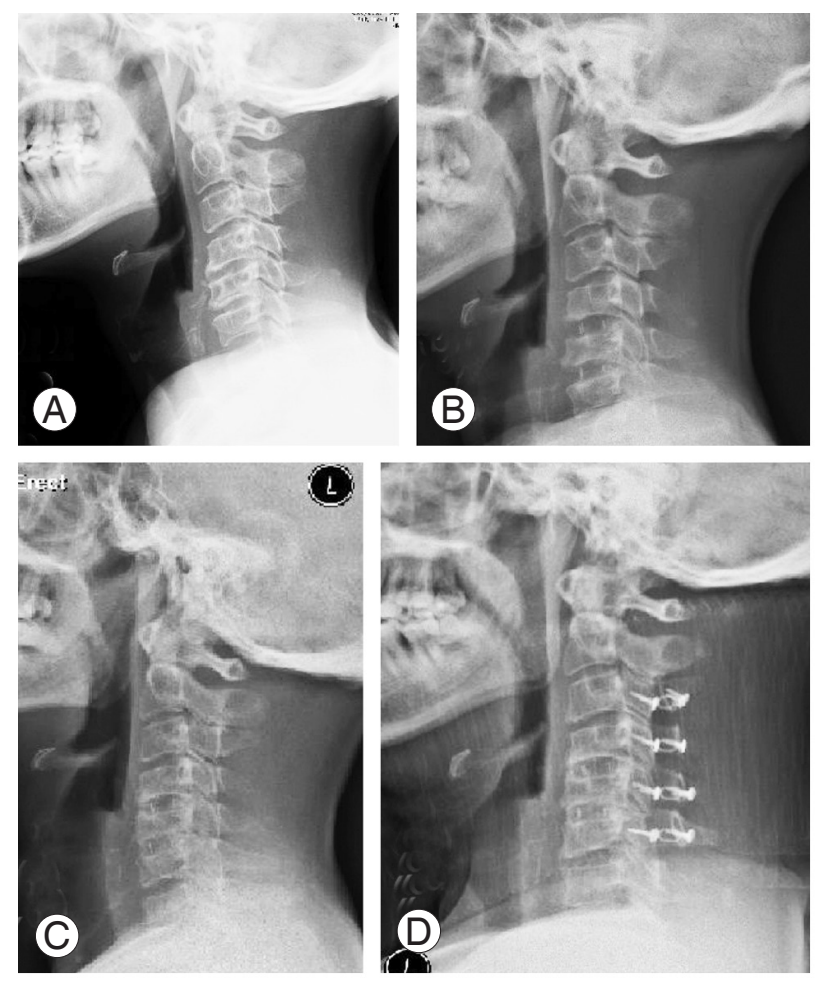

Fig. 3. Adjacent segment disease after C4-6 ACDF. (A) Preoperative lateral X-ray. (B) Postoperative X-ray of C4-6 ACDF. (C) X-ray at 3 years demonstrating adjacent segment disease. (D) Postoperative film of C3-6 laminoplasty. ACDF, anterior cervical discectomy and fusion.

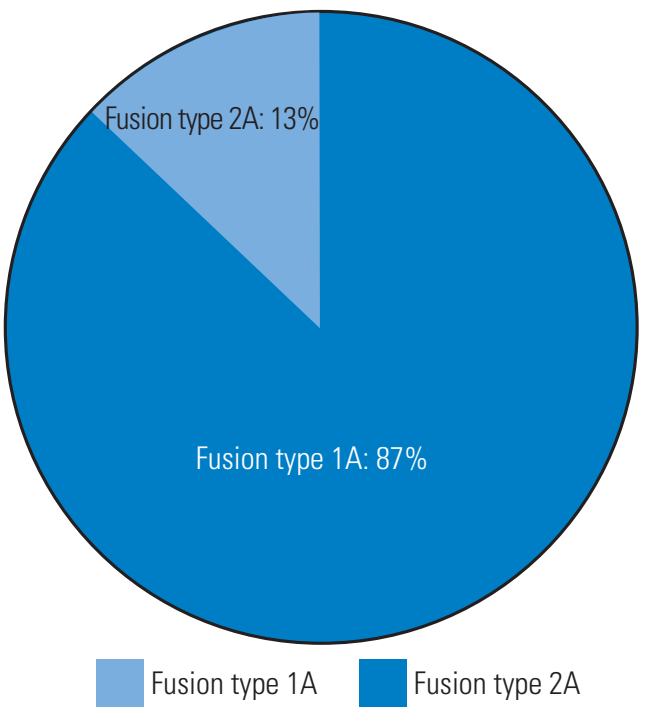

Fig. 4. Type of fusion achieved at 12 months postoperatively.

total, 27 patients (87\%) achieved type 1A fusion and four (13\%) achieved type 2A fusion (Fig. 4).

In total, 14 cages (22.5\%) developed radiological cage subsidence at 6 months. Of these, 11 cages $(78.5 \%)$ had subsidence between 3 and $5 \mathrm{~mm}$, and three (12.5\%) had ra- 
Table 3. Characteristics of patients with subsidence

\begin{tabular}{|c|c|c|c|}
\hline Characteristic & Subsidence & No subsidence & p-value \\
\hline Age (yr) & $62.4 \pm 14.8$ & $59.0 \pm 11.8$ & 0.522 \\
\hline Male sex & $6(85.7)$ & 19 (79.2) & 0.587 \\
\hline Level of fusion & & & 0.450 \\
\hline C3-5 & 5 & 9 & \\
\hline C4-7 & 2 & 8 & \\
\hline $\mathrm{C5}-8$ & 0 & 6 & \\
\hline \multicolumn{4}{|c|}{ Japanese Orthopaedic Association score } \\
\hline Preoperative & $10.9 \pm 2.0$ & $9.80 \pm 2.3$ & 0.283 \\
\hline $3 \mathrm{mo}$ & $13.8 \pm 2.6$ & $13.0 \pm 2.0$ & 0.522 \\
\hline $6 \mathrm{mo}$ & $13.0 \pm 2.8$ & $13.4 \pm 2.1$ & 0.814 \\
\hline $1 \mathrm{yr}$ & $13.0 \pm 2.8$ & $13.4 \pm 2.2$ & 0.841 \\
\hline $2 \mathrm{yr}$ & $13.0 \pm 2.8$ & $13.5 \pm 2.0$ & 0.617 \\
\hline
\end{tabular}

Values are presented as mean \pm standard deviation or number (\%).

diological subsidence of more than $5 \mathrm{~mm}$. There were no additional cages with subsidence during the subsequent follow-ups at 6 months to 2 years. Interestingly, there was no statistically significant difference when comparing the JOA scores between patients with cage subsidence and those without it at the preoperative baseline $(p=0.283), 3$ months $(p=0.522), 6$ months $(p=0.814), 1$ year $(p=0.841)$, and 2 years $(p=0.126)$. There was also no significant association between subsidence and age $(p=0.522)$, sex ( $p=0.587)$, and the levels of fusion $(p=0.450)$, as detailed in Table 3.

The mean preoperative $\mathrm{C} 2-7$ angle was $8.19^{\circ} \pm 13.2^{\circ}$. The postoperative 3 -month $\mathrm{C} 2-7$ angle was $10.7^{\circ} \pm 11.5^{\circ}$ $(p=0.354)$, postoperative 6-month angle was $11.5^{\circ} \pm 14.1^{\circ}$ $(p=0.937)$, postoperative 1-year angle was $13.0^{\circ} \pm 15.3^{\circ}$ $(p=0.367)$, and the postoperative 2 -year angle was $10.9^{\circ} \pm 14.5^{\circ}(p=0.201)$ (Table 2). Although there was a lordotic change of the mean $\mathrm{C} 2-7$ angle up to 2 years after ACDF, the difference did not reach statistical significance.

The mean preoperative LSA was $4.44^{\circ} \pm 8.91^{\circ}$. The postoperative 3 -month LSA was $5.56^{\circ} \pm 6.79^{\circ}(p=0.451)$, postoperative 6 - month LSA was $5.76^{\circ} \pm 8.61^{\circ}(p=0.86)$, postoperative 1-year LSA was $5.29^{\circ} \pm 10.3^{\circ}(p=0.741)$, and postoperative 2 -year LSA was $3.06^{\circ} \pm 9.03^{\circ}(p=0.198)$, as shown in Table 2. Although a lordotic change in the LSA after ACDF was observed up to 1 year of follow-up, the change was not statistically significant. At the 2-year follow-up, there was a loss of lordosis in the LSA when compared to preoperatively, yet the difference was not statisti- cally significant. Fusion segmental kyphosis of more than $5^{\circ}$ occurred in one patient $(3.2 \%)$. There were no cases of cage migration in this series.

\section{Discussion}

ACDF is one of the most commonly performed operative procedures for cervical degenerative diseases. This technique initially involved the use of autologous iliac crest bone grafts, which soon became associated with significant donor site morbidity. Newer techniques using locking stand-alone cages or an anterior plate construct have replaced the earlier procedure and eliminated the morbidity associated with iliac crest bone graft harvesting. Use of the stand-alone cage construct eliminates complications related to the plate, such as screw loosening, pull-out, and foreign body sensation or dysphagia [9].

The safety of locking stand-alone cages in single-level ACDF has been established in various case studies. The meta-analysis by Nambiar et al. [6] in 2017 noted that a single-level ACDF with cage-only constructs achieved similar functional outcomes and radiological parameters, such as segmental Cobb's angle, cervical lordosis, and fusion rates, when compared to cages with plate constructs. There was, however, a paucity of evidence evaluating the use of the stand-alone cage approach in 2-level ACDF procedures.

In our current study, patients who underwent 2-level ACDF with stand-alone cages had significantly improved 
JOA scores, when compared to those before surgery. The mean recovery rate in the JOA was $43.9 \%$. These improvements persisted throughout subsequent followups to at least 2 years after the initial operation. However, two patients (6.4\%) developed ASD requiring a second operation. In comparison, Song et al. [10] and Joo et al. [11] reported rates of radiological ASD of $7.9 \%$ and $4.5 \%$, respectively, for 2-level stand-alone cage constructs in their studies, although no revision surgery was required to address these complications. Reported risk factors for ASD include the presence of ASD before surgery [12], increased range of motion in the adjacent level, and excessive disk space distraction [13]. However, the debate on the exact etiology of ASD continues; it is so far unclear whether it is due to the natural history of cervical spondylosis or to postoperative changes, such as subsidence, changes in disc height, or changes in cervical alignment.

In this study, we found a subsidence rate of $22.5 \%$ for the 2-level stand-alone cage construct, which occurred at 6 months of follow-up and remained static until the final follow-up at 2 years. In the literature on 2-level ACDF, this subsidence rate has been shown to vary substantially. For example, Kim et al. [14] showed a subsidence rate of $66.6 \%$ at 24 months of follow-up in their recent prospective study in which 12 patients received 2-level stand alone cage construct. In retrospective studies of 2-level ACDF using a cage-only construct, Joo et al. [11] reported a subsidence rate of $31.8 \%$, and $\mathrm{Oh}$ et al. [15] reported a subsidence rate of $35.7 \%$. For single-level ACDF, the subsidence rate reported regarding the use of stand-alone PEEK cages in the literature has also ranged considerably, from $8.1 \%$ [16] to $56 \%$ [8], and the mean incidence of subsidence using PEEK cages in literature is $23.5 \%$ [17].

Cage subsidence in ACDF has been shown to be affected by various operative factors. Bartels et al. [18] demonstrated that the fusion level of C6-7 had a higher incidence of cage subsidence than other fusion levels. Barsa and Suchomel [19] reported subsidence to be associated with a greater distance from the anterior rim of the upper vertebra to the cage, and to a smaller contact surface ratio of the cage. Yang et al. [20] identified the use of smaller anteroposterior diameter cages as well as larger intraoperative distractions associated with a higher incidence of subsidence. In our study, subsidence was not shown to be affected by age, sex, or the level of fusion. Our patients who experienced cage subsidence had similar JOA scores when compared to patients who had no subsidence, suggesting subsidence may not affect clinical outcomes. Although subsidence may lead to intervertebral foramen narrowing, resulting in compression of the exiting nerve roots, evidence in the literature regarding whether this risk has clinical significance is still inadequate [14].

This study yielded a fusion rate of $100 \%$ at 12 months of follow-up. The literature has documented satisfactory fusion rates in 2-level ACDF, using a cage-only construct, ranging from $83.3 \%$ [10] to $90.9 \%$ [11].

Although we noted a lordotic change of cervical global alignment, as measured by the $\mathrm{C} 2-7$ angle and LSA following ACDF up to 1 year, the change observed did not reach statistical significance. Subsequently, at 2 years, we noted a slight loss of lordosis of the mean $\mathrm{C} 2-7$ angle and the LSA when compared to the values at 1 year. In addition, the LSA at 2 years of $3.06^{\circ} \pm 9.03^{\circ}$ was even less lordotic than the preoperative value of $4.44^{\circ} \pm 8.91^{\circ}$. This phenomenon was also observed in the study by Yang et al. [20], who recorded an initial lordotic change in the LSA after surgery, which became kyphotic at the last follow-up. Barsa and Suchomel [19] reported an $8.7^{\circ}$ loss of segmental lordosis using Cobb's angle at 6 months of follow-up for single-level stand-alone cage procedures, while Han et al. [21] recorded a mean change of $1.08^{\circ}$ in kyphosis at 12 months. For 2-level cage-only ACDF, Kim et al. [14] reported a kyphotic change of both the $\mathrm{C} 2-7$ angle and the LSA at 24 months of follow-up. The reasons for the loss of lordosis or kyphotic change in the locally fused segment postoperatively were commonly attributed to disc degeneration with loss of disc height, adjacent segment kyphosis, or cage subsidence.

This study represents mid-term results for 2-level ACDF procedures in a regional center and is predominantly limited by its small sample size and retrospective nature. The sample size compromises the power of the study and reduced our ability to detect significant differences, especially in cervical alignment measurements. In the future, prospective studies with larger sample sizes and longer follow-ups are warranted to provide more solid data on the long-term effects of using 2-level stand-alone cages. In addition, a greater battery of clinical parameters, including cervical range of motion, Visual Analogue Scale score, and the Neck Disability Index, could be productively utilized in future studies. 


\section{Conclusions}

This study demonstrated that stand-alone PEEK cages can improve clinical outcomes and help achieve satisfactory fusion in 2-level ACDF procedures.

\section{Conflict of Interest}

No potential conflict of interest relevant to this article was reported.

\section{References}

1. Smith GW, Robinson RA. The treatment of certain cervical-spine disorders by anterior removal of the intervertebral disc and interbody fusion. J Bone Joint Surg Am 1958;40-A:607-24.

2. Silber JS, Anderson DG, Daffner SD, et al. Donor site morbidity after anterior iliac crest bone harvest for single-level anterior cervical discectomy and fusion. Spine (Phila Pa 1976) 2003;28:134-9.

3. Riley LH 3rd, Skolasky RL, Albert TJ, Vaccaro AR, Heller JG. Dysphagia after anterior cervical decompression and fusion: prevalence and risk factors from a longitudinal cohort study. Spine (Phila Pa 1976) 2005;30:2564-9.

4. Kapetanakis S, Thomaidis T, Charitoudis G, Pavlidis P, Theodosiadis P, Gkasdaris G. Single anterior cervical discectomy and fusion (ACDF) using self-locking stand-alone polyetheretherketone (PEEK) cage: evaluation of pain and health-related quality of life. J Spine Surg 2017;3:312-22.

5. Overley SC, Merrill RK, Leven DM, Meaike JJ, Kumar A, Qureshi SA. A matched cohort analysis comparing stand-alone cages and anterior cervical plates used for anterior cervical discectomy and fusion. Global Spine J 2017;7:394-9.

6. Nambiar M, Phan K, Cunningham JE, Yang Y, Turner PL, Mobbs R. Locking stand-alone cages versus anterior plate constructs in single-level fusion for degenerative cervical disease: a systematic review and meta-analysis. Eur Spine J 2017;26:2258-66.

7. Peolsson A, Hedlund R, Vavruch L. Prediction of fusion and importance of radiological variables for the outcome of anterior cervical decompression and fusion. Eur Spine J 2004;13:229-34.

8. Gercek E, Arlet V, Delisle J, Marchesi D. Subsidence of stand-alone cervical cages in anterior interbody fusion: warning. Eur Spine J 2003;12:513-6.

9. Pereira EA, Chari A, Hempenstall J, Leach JC, Chandran H, Cadoux-Hudson TA. Anterior cervical discectomy plus intervertebral polyetheretherketone cage fusion over three and four levels without plating is safe and effective long-term. J Clin Neurosci 2013;20:1250-5.

10. Song KJ, Taghavi CE, Lee KB, Song JH, Eun JP. The efficacy of plate construct augmentation versus cage alone in anterior cervical fusion. Spine (Phila $\mathrm{Pa}$ 1976) 2009;34:2886-92.

11. Joo YH, Lee JW, Kwon KY, Rhee JJ, Lee HK. Comparison of fusion with cage alone and plate instrumentation in two-level cervical degenerative disease. J Korean Neurosurg Soc 2010;48:342-6.

12. Song KJ, Choi BW, Kim JK. Adjacent segment pathology following anterior decompression and fusion using cage and plate for the treatment of degenerative cervical spinal diseases. Asian Spine J 2014;8:720-8.

13. Li J, Li Y, Kong F, Zhang D, Zhang Y, Shen Y. Adjacent segment degeneration after single-level anterior cervical decompression and fusion: disc space distraction and its impact on clinical outcomes. J Clin Neurosci 2015;22:566-9.

14. Kim SY, Yoon SH, Kim D, Oh CH, Oh S. A prospective study with cage-only or cage-with-plate fixation in anterior cervical discectomy and interbody fusion of one and two levels. J Korean Neurosurg Soc 2017;60:691-700.

15. Oh JK, Kim TY, Lee HS, et al. Stand-alone cervical cages versus anterior cervical plate in 2-level cervical anterior interbody fusion patients: clinical outcomes and radiologic changes. J Spinal Disord Tech 2013;26:415-20.

16. Ha SK, Park JY, Kim SH, Lim DJ, Kim SD, Lee SK. Radiologic assessment of subsidence in stand-alone cervical polyetheretherketone (PEEK) cage. J Korean Neurosurg Soc 2008;44:370-4.

17. Noordhoek I, Koning MT, Jacobs WC, VleggeertLankamp CL. Incidence and clinical relevance of cage subsidence in anterior cervical discectomy and fusion: a systematic review. Acta Neurochir (Wien) 2018;160:873-80.

18. Bartels RH, Donk R, van Azn RD. Height of cervical foramina after anterior discectomy and implantation of a carbon fiber cage. J Neurosurg 2001;95(1 
Suppl):40-2.

19. Barsa P, Suchomel P. Factors affecting sagittal malalignment due to cage subsidence in standalone cage assisted anterior cervical fusion. Eur Spine J 2007;16:1395-400.

20. Yang JJ, Yu CH, Chang BS, Yeom JS, Lee JH, Lee CK.
Subsidence and nonunion after anterior cervical interbody fusion using a stand-alone polyetheretherketone (PEEK) cage. Clin Orthop Surg 2011;3:16-23.

21. Han SY, Kim HW, Lee CY, Kim HR, Park DH. Standalone cages for anterior cervical fusion: are there no problems? Korean J Spine 2016;13:13-9. 